\title{
A NOTE ON MATRICES DEFINING TOTAL REAL FIELDS*
}

\author{
BY A. A. ALBERT
}

Let $K$ be algebraic of degree $n$ over a sub-field $F$ of the field of all real numbers. Then there is an equation

$$
f(x)=x^{n}+a_{1} x^{n-1}+\cdots+a_{n}=0, \quad\left(a_{i} \text { in } F\right),
$$

which is irreducible in $F$, and $K=F(X)$ consists of all polynomials with coefficients in $F$ in an algebraic quantity $X$ for which $f(x)=0$. We call $K$ a total real field over $F$ if the ordinary complex roots

$$
x_{1}, \cdots, x_{n}
$$

of $f(x)=0$ are all real. The modern theory of algebraic numbers has made the study of such fields of great interest.

A particular algebraic root of $f(x)=0$ is given by the matrix

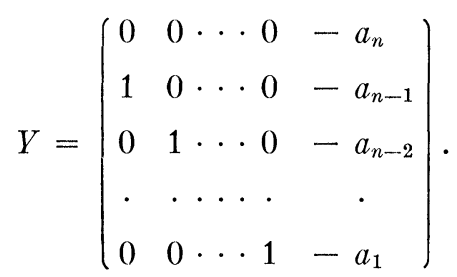

This is a matrix whose characteristic equation is the above $f(x)=0$. The irreducibility of $f(x)$ implies that every $n$-rowed square matrix $Z$ with elements in $F$ and $f(x)=0$ as characteristic equation is similar to $Y$, and thus every such $Z$ defines a field $F(Z)$ equivalent to $K$ over $F$.

We shall obtain a normal form here for $Z$ such that every $Z$ in our form and with irreducible characteristic equation defines a total real field, while conversely every total real field is defined by one of our matrices. Our result will then provide a construction of all total real fields over $F$. The irreducibility condition is of course a part of the final conditions in all problems on the construction of algebraic fields and should not be considered as

* Presented to the Society, December 31, 1936. 
affecting the completeness of our criterion. We shall in fact prove the following theorem.

TheOREM. Let $D$ be an n-rowed diagonal matrix with positive diagonal elements in $F$, and $S$ be any symmetric n-rowed square matrix with elements in $F$ for which the characteristic function of

$$
Z=D S
$$

is irreducible in $F$. Then $F(Z)$ is a total real field of degree $n$ over $F$. Conversely every total real field $K$ of degree $n$ over $F$ is equivalent to a field $F(Z)$ with $Z$ given by (4).

For if $D$ and $E$ are the $n$-rowed diagonal matrices*

(5) $D=\operatorname{diag}\left\{d_{1}, \cdots, d_{n}\right\}, e_{i}=d_{i}{ }^{1 / 2}, E=\operatorname{diag}\left\{e_{1}, \cdots, e_{n}\right\}$,

then $D=E^{2}, E=E^{\prime}$ is a real symmetric matrix,

$$
E^{-1} Z E=E^{-1} E^{2} S E=E S E^{\prime}
$$

is a real symmetric matrix. Thus the characteristic roots of $E^{-1} Z E$ are all real. But they are the roots of the characteristic equation of $Z$ and we are assuming that this equation is irreducible in $F$. Hence $F(Z)$ is a total real field.

Conversely let $K$ be total real of degree $n$ over $F$ so that $K$ is equivalent over $F$ to $F(Y)$ with $Y$ given by (3). We let $V$ be the Vandermonde matrix

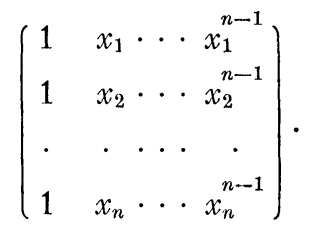

The square of the determinant of $V$ is the discriminant of $f(x)$ and is not zero when $f(x)$ is irreducible in $F$. This is our hypothesis, so that the matrix

$$
T=V^{\prime} V=\left(s_{i+j-2}\right), \quad(i, j=1, \cdots, n),
$$

is non-singular. Also the symmetric function $s_{k}=\sum_{g=1}^{n} x_{g}^{k}$ is well known to be a polynomial in $a_{1}, \cdots, a_{n}$ with integral coeffi-

* We use the notation $\operatorname{diag}\left\{d_{1}, \cdots, d_{n}\right\}$ to mean an $n$-rowed square matrix whose elements off the principal diagonal are zero and whose principal diagonal is $d_{1}, \cdots, d_{n}$. 
cients, so that $T$ has elements in $F$. Since $V$ is a real non-singular matrix, the matrix $T=V^{\prime} V$ is positive definite symmetric. This is actually the true reason for our result.*

There exists a non-singular $B$ with elements in $F$ such that

$$
B^{\prime} T B=\left(\begin{array}{llll}
g_{1} & & & \\
& \cdot & & \\
& \cdot & \\
& & & \\
& & & g_{n}
\end{array}\right), \quad\left(g_{i} \text { in } F^{\prime}\right) .
$$

Since $T$ is positive definite, so is $B^{\prime} T B$, and the $g_{i}$ must be positive. Thus

$$
D=\operatorname{diag}\left\{d_{1}, \cdots, d_{n}\right\}, \quad d_{i}=g_{i}^{-1}>0, D^{-1}=B^{\prime} T B .
$$

By an elementary computation

$$
V Y V^{-1}=\operatorname{diag}\left\{x_{1}, \cdots, x_{n}\right\} .
$$

The diagonal matrix $V Y V^{-1}$ is symmetric and

$$
\left(V Y V^{-1}\right)^{\prime}=\left(V^{\prime}\right)^{-1} Y^{\prime} V^{\prime}=V Y V^{-1}, \quad\left(V^{\prime} V\right) Y=Y^{\prime}\left(V^{\prime} V\right) .
$$

Hence $T Y=Y^{\prime} T,\left(B^{\prime} T B\right) B^{-1} Y B=B^{\prime} Y^{\prime}\left(B^{\prime}\right)^{-1} B^{\prime} T^{\prime} B$, whence

$$
D^{-1} Z=Z^{\prime}\left(D^{-1}\right)^{\prime}, \quad Z=B^{-1} Y B .
$$

The matrix $S=D^{-1} Z$ is now symmetric since $S^{\prime}=Z^{\prime}\left(D^{-1}\right)^{\prime}=S$. Then

$$
Z=D S
$$

as desired, and our theorem is proved.

Notice in closing that the positive elements of the matrix $D$ are the inverses of the elements in the diagonal normal form of the discriminant matrix $T$. When this normal form of $T$ is the identity matrix the result $Z$ is a symmetric matrix $S$ for which the total reality of $F(Z)$ is a classical result. $\dagger$

The University of Chicago

* See Bieberbach-Bauer, Vorlesungen über Algebra, 1933, p. 184, for the known theorem stating that $T$ is positive definite when $f(x)=0$ has all real roots. That this result is true is an evident consequence of the definition of positive definiteness.

$\dagger$ See L. E. Dickson, Modern Algebraic Theories, 1926, p. 76; Theorem 12. 